\title{
Susceptibility to $\beta$-lactam antibiotics of mutant strains of Xanthomonas maltophilia with high- and low-level constitutive expression of $\mathrm{L} 1$ and $\mathrm{L} 2 \beta$-lactamases
}

\author{
M. AKOVA, G. BONFIGLIO and D. M. LIVERMORE*
}

Department of Medical Microbiology, The London Hospital Medical College, Tüner Street, London E1 2AD

\begin{abstract}
Summary. Xanthomonas maltophilia produces two inducible $\beta$-lactamases, L1 and L2, and resists the antimicrobial activity of $\beta$-lactam antibiotics, including carbapenems. $L 1$ is a zinc-metaloenzyme with carbapenemase activity; L2 is an unusual cephalosporinase. Mutant strains with high-and low-level constitutive expression of these enzymes were derived from three reference strains of $X$. maltophilia. With a single exception, the mutant strains had altered expression of both enzymes, indicating that these $\beta$-lactamases share regulatory components. The exception was a mutant strain that had low-level constitutive (basal) expression of $\mathrm{L} 1$ enzyme but remained inducible for $\mathrm{L} 2$. A parent strain with low-level $\beta$ lactamase inducibility was more susceptible to penicillins, cephalosporins and carbapenems than were those in which higher levels of enzyme activity were inducible. Mutations that caused high-level constitutive $\beta$-lactamase expression increased resistance to penicillins and newer cephalosporins. $\beta$-Lactamase basal mutant strains, including the one that remained inducible for L2 enzyme, were more susceptible than inducible strains to these drugs. Organisms with inducible or high-level constitutive $\beta$-lactamase expression were equally resistant to meropenem and imipenem but basal mutant strains, including the one that remained inducible for $\mathbf{L} 2$ enzyme, were more susceptible to meropenem than imipenem. Minimal inhibitory concentrations of meropenem, penicillins and cephalosporins, but not imipenem, were greater on Mueller Hinton agar than on IsoSensitest or Diagnostic Sensitivity Test agars. This behaviour was independent of $\beta$-lactamase inducibility, and may reflect permeability differences between cells grown on different media.
\end{abstract}

\section{Introduction}

Xanthomonas maltophilia, previously known as Pseudomonas maltophilia, has become a focus of interest because of the increasing frequency of its isolation from patients, and its broad-spectrum antimicrobial resistance. ${ }^{1}$ Severe infections, including endocarditis, meningitis and septicaemia, may be caused by this organism in seriously ill or immunocompromised patients. More often, however, the organism is isolated from mixed infections, in which its pathogenic role is debatable. $X$. maltophilia has almost no ability to cause infection after injection into healthy animals. ${ }^{2}$ The emergence of the organism as a pathogen has been attributed to selective pressure by use of broadspectrum antibiotics ${ }^{3}$ but may also reflect the increasingly large numbers of patients who are sufficiently ill to be susceptible to infection.

Received 11 Dec. 1990; accepted 11 Jan. 1991.

* Correspondence should be sent to Dr D. M. Livermore.
Few antimicrobial agents are effective against $X$. maltophilia and it is notorious for its resistance to $\beta$ lactam antibiotics, including carbapenems. ${ }^{4}$ It has been suggested that low outer-membrane permeability and production of two different chromosomallymediated $\beta$-lactamases, L1 and L2, contribute to this resistance. $^{5-7}$ Both enzymes are inducible. $\mathrm{Ll}$ is a unique tetrameric penicillinase with a zinc ion in its active site and is capable of hydrolysing carbapenems such as meropenem and imipenem. ${ }^{8}$ L2 is a cephalosporinase with a broad substrate profile; it hydrolyses penicillins and aztreonam as well as cephalosporins. ${ }^{9}$ Unlike L1 enzyme, it is inhibited by clavulanate and sulbactam. ${ }^{6}$ In view of the wide substrate range of this combination of $\beta$-lactamases, their contribution to resistance has been assumed to be of major importance. However, this view has not been confirmed by comparative susceptibility tests on strains with mutations in the genes controlling $\beta$-lactamase production, except that mutant strains with high-level constitutive expression of the enzymes have been reported to show 
slightly increased resistance, compared with their $\beta$ lactamase-inducible parent strains. ${ }^{7}$

It has been noted also that the minimum inhibitory concentrations (MICs) of $\beta$-lactam antibiotics for $X$. maltophilia isolates vary with the test medium used. ${ }^{10,11}$ This may be related to the induction of $\beta$ lactamases. Medium-dependency occurs with induction of Class I $\beta$-lactamases in a few strains of enterobacteria and $P$. aeruginosa ${ }^{12}$ but has not been investigated for $X$. maltophilia.

We have obtained strains with mutations in genes controlling $\beta$-lactamase inducibility from three $X$. maltophilia strains. The mutant strains constitutively expressed either high or low levels of $\mathrm{L} 1$ and $\mathrm{L} 2$ $\beta$-lactamases. The antibiotic susceptibility of these mutant strains was compared with that of their $\beta$ lactamase-inducible parent strains to reveal the relationship between $\beta$-lactamase expression and resistance. The mutant strains also were used to establish whether medium-dependency of $\beta$-lactamase induction was a source of variation in MIC.

\section{Materials and methods}

\section{Derivation of mutant strains}

$X$. maltophilia strains NCTC 10257, NCTC 10258 and NCTC 10499 were used; all were inducible for both $\mathrm{L} 1$ and $\mathrm{L} 2 \beta$-lactamases. To derive mutant strains with high-level constitutive (stably de-repressed) $\beta$ lactamase expression, exponential phase cultures were treated for 30-60 min with the mutagens $\mathrm{N}-$-methyl $\mathrm{N}^{\prime}-$ nitro N-nitroso-guanidine (NTG) $100 \mathrm{mg} / \mathrm{L}$ or ethyl methane sulphonate (EMS) $0.5 \% \mathrm{v} / \mathrm{v}$. After mutagenesis, the cells were harvested and washed in $0.1 \mathrm{M}$

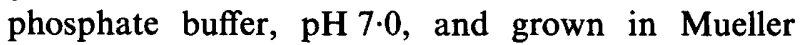
Hinton Broth (Oxoid CM405) for 2-13 h, to allow expression of mutations, before plating on to Mueller Hinton Agar (Oxoid CM337) containing mezlocillin, piperacillin or cefoperazone at $4-8 \times$ MIC for the parent strain. Colonies that reached $>2 \mathrm{~mm}$ in diameter after overnight incubation were subcultured, and part of the colony was emulsified in $0.1 \mathrm{M}$

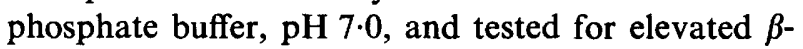
lactamase expression with $1 \mathrm{mM}$ nitrocefin prepared in the same buffer. Rapid development of a red colour indicated that the colony hyperproduced $\beta$-lactamase. Mutant strains with low-level constitutive (basal) $\beta$ lactamase expression were also derived by mutagenesis with NTG or EMS, as above. They were detected by overnight culture of the treated cells on antibiotic-free nutrient agar and then flooding the plates with $80 \%$ strength Mueller Hinton agar containing benzylpenicillin $6 \mathrm{~g} / \mathrm{L}$, iodine $0.2 \% \mathrm{w} / \mathrm{v}$ and potassium iodide $2 \% \mathrm{w} / \mathrm{v} .{ }^{13}$ Colonies of $\beta$-lactamase-inducible cells, or those with high-level constitutive expression, gave a clear halo due to reduction of the iodine by reaction with the hydrolysis product of penicillin. $\beta$-Lactamase basal mutant strains did not give a halo.

\section{Antibiotics and other reagents}

Ampicillin and carbenicillin were obtained from SmithKline Beecham (Brockham Park, Surrey); aztreonam from E. R. Squibb and Sons (Princeton, NJ, USA), ceftazidime from Glaxo Group Research (Greenford, Middlesex); cefsulodin from Ciba-Geigy Pharmaceuticals (Horsham, Sussex); imipenem from Merck Sharp and Dohme (Hoddesdon, Herts); latamoxef (moxalactam) from Eli Lilly \& Co Ltd (Basingstoke, Hants); meropenem (SM-7338) from ICI Pharmaceuticals (Macclesfield, Cheshire); piperacillin from Lederle Laboratories (Gosport, Hants); and chloramphenicol from Parke Davis (Pontypool, Gwent). Other antimicrobial agents and other chemical reagents were obtained from the Sigma Chemical Co., (Poole, Dorset).

\section{Induction assays}

Bacteria were grown routinely overnight in Mueller Hinton broth at $37^{\circ} \mathrm{C}$ with continuous shaking. In experiments to determine the effect of the medium on $\beta$-lactamase induction, IsoSensitest Broth (Oxoid CM473) or a broth equivalent to Diagnostic Sensitivity Test (DST) Agar (Oxoid CM261) were used.

The overnight cultures were diluted 10 -fold into pre-warmed broth from the same batch and incubated for a further $90 \mathrm{~min}$. Cefotaxime, one of the most potent inducers of $\beta$-lactamase in $X$. maltophilia, ${ }^{7}$ was then added at $0 \cdot 25-4 \times$ MIC. After $2 \mathrm{~h}$, the cells were harvested, washed, and their $\beta$-lactamase(s) released by sonication. The enzyme activity was measured, by UV spectrophotometry, against $0.1 \mathrm{~mm}$ imipenem at $297 \mathrm{~nm}$, and against $0.1 \mathrm{mM}$ cefsulodin at $263 \mathrm{~nm}$. Imipenem is a specific substrate for Ll enzyme and cefsulodin for L2 enzyme. ${ }^{5-7}$ The light path was $1 \mathrm{~cm}$, temperature $37^{\circ} \mathrm{C}$ and the assay buffer was $0.1 \mathrm{~mm}$ sodium phosphate, $\mathrm{pH} \mathrm{7.0.} \mathrm{The} \mathrm{assay} \mathrm{mixture} \mathrm{was}$ supplemented with $0 \cdot 1 \mathrm{~mm}$ zinc sulphate when imipenem was the substrate, because of the suggestion that the $\mathrm{L} 1$ enzyme is unstable below $\mathrm{pH} 8$ unless zinc ions are present. ${ }^{8}$ The mixture was supplemented with $0.1 \mathrm{~mm}$ edetic acid, but not zinc sulphate, when cefsulodin was the substrate, to inactivate Ll enzyme and allow precise determination of $\mathrm{L} 2$ activity. Enzyme yields were standardised against protein concentration, as measured by the method of Lowry et al. ${ }^{14}$

\section{Iso-electric focusing}

The iso-electric points of $\beta$-lactamases were determined by iso-electric focusing. ${ }^{15}$

\section{Susceptibility tests}

MICs of $\beta$-lactams and other antibiotics were determined on Mueller Hinton, IsoSensitest and DST agars by an agar dilution method. The inoculum was 


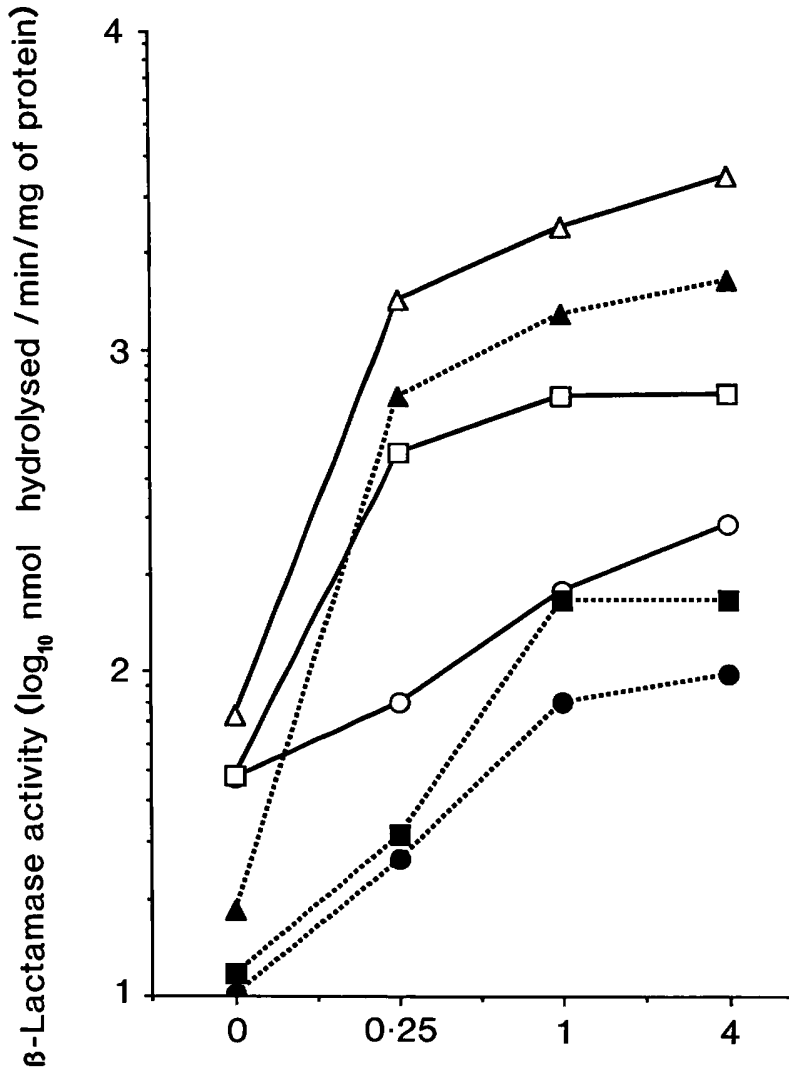

Cefotaxime concentration factor (x MIC)

Fig. 1. $\beta$-Lactamase induction by cefotaxime in $X$. maltophilia strains NCTC $10257(\triangle, \triangle)$, NCTC $10258(\bullet, O)$ and NCTC $10499(\square$, $\square)$. The open symbols indicate activity against imipenem, the closed symbols that against cefsulodin.

$10^{4} \mathrm{cfu}$ taken from an overnight growth in the corresponding broth medium. MICs were read as the lowest antibiotic concentrations that prevented visible growth after incubation for $24 \mathrm{~h}$ at $37^{\circ} \mathrm{C}$.

\section{Results}

\section{$\beta$-Lactamase expression and derivation of mutant strains}

$\beta$-Lactamase activity against imipenem and cefsulodin was inducible with cefotaxime in all three parent strains (fig. 1), although the levels induced in NCTC 10258 were lower than those in the other two strains. The derivation of $\beta$-lactamase-hyperproducing and basal mutant strains proved considerably more difficult with $X$. maltophilia strains than had been our previous experience with $P$. aeruginos $a$ and with various enterobacteria. ${ }^{13,16}$ Spontaneous mutant strains with highlevel constitutive $\beta$-lactamase expression could not be selected on agar containing $\beta$-lactams and were derived only after cultures had been exposed to NTG or EMS. It was also found that $\beta$-lactamase-inducible organisms, as well as those with high-level constitutive production, gave a positive reaction on plates flooded with the penicillin-iodine mixture. With enterobacteria and $P$. aeruginosa, only high-level constitutive ("stably de-repressed") producers of Class I enzymes showed this behaviour. ${ }^{13,16}$

Nevertheless, mutant strains were obtained with high- and low-level constitutive $\beta$-lactamase expression from all three strains. Mutant strains of these types (table I) were designated by the suffixes -con and -def, respectively. Iso-electric focusing of induced and uninduced extracts, and hydrolysis assays with specific substrates, indicated that expression of both $\mathrm{L} 1$ (pI 6.5 , active against imipenem but not cefsulodin) and L2 (pI 8.5, active against cefsulodin but not imipenem) enzymes was affected simultaneously in all but one of the mutant strains. The exception was mutant strain 7-L1-def, which had basal activity against imipenem but retained inducible $\beta$-lactamase activity against cefsulodin. Two different $\beta$-lactamase constitutive

Table I. $\beta$-Lactamase expression mutant strains of $X$. maltophilia

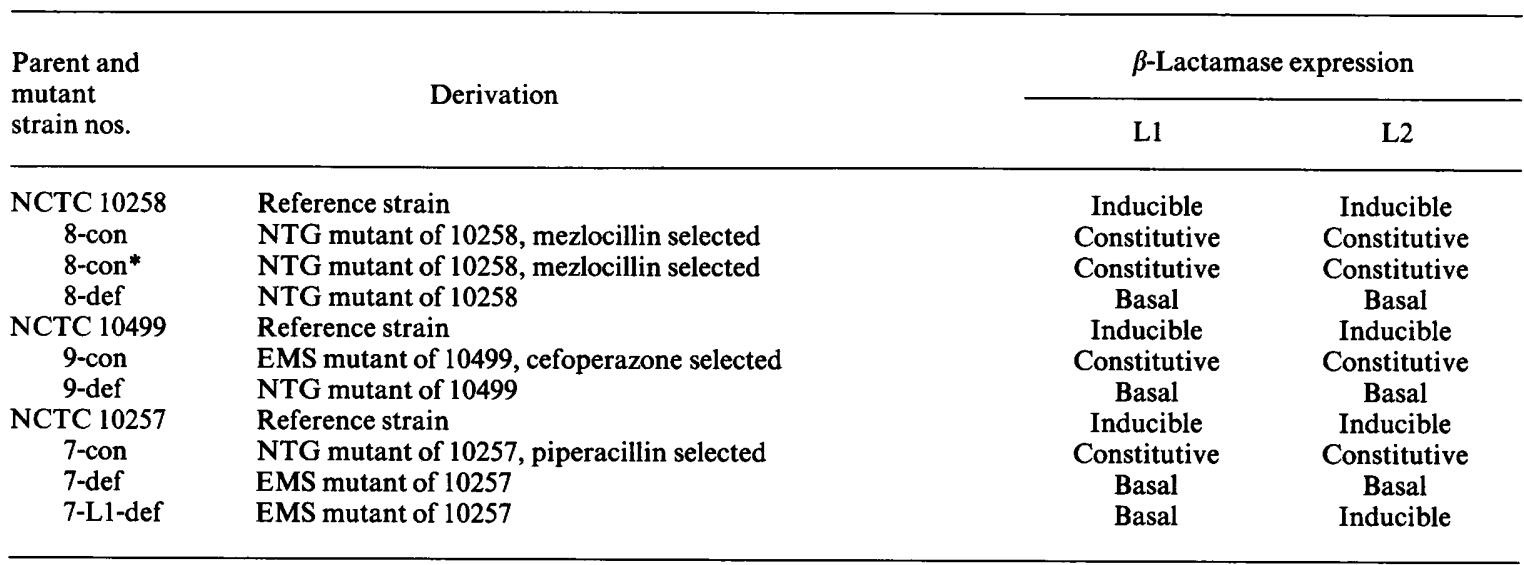

$\beta$-Lactamase-inducible strains produced low levels of activity in the absence of inducers, but high levels in their presence; induction ratios (amount of enzyme with inducer/amount of enzyme without inducer) were $\geq 10$. $\beta$-Lactamase constitutive organisms had high levels of enzyme and basal ones low levels, irrespective of the presence of inducer; apparent induction ratios were $\leq 2$. Numerical estimates of $\beta$-lactamase expression are not given because the hydrolysis traces for both compounds tended to be non-linear; the rate initially declined more rapidly than was explicable by substrate depletion and then stabilised. This behaviour, which may indicate enzyme deactivation, distorted accurate calculation of hydrolysis rates but did not preclude gross characterisation of the inducibility behaviour. 
Table II. MICs of $\beta$-lactam agents for the inducibility mutant strains of $X$. maltophilia on Mueller Hinton agar

\begin{tabular}{|c|c|c|c|c|c|c|c|c|c|c|c|}
\hline \multirow{2}{*}{ Strain } & \multicolumn{11}{|c|}{$\mathrm{MIC}(\mathrm{mg} / \mathrm{L})$ of } \\
\hline & IMP & MEM & AMP & PIP & CARB & CEF & CTX & CTZ & CPZ & LMX & $\mathrm{AZT}$ \\
\hline NCTC 10258 & 64 & 4 & 4 & 16 & 8 & 4 & 8 & 2 & 4 & 4 & 16 \\
\hline 8-def & 4 & 0.5 & 1 & 4 & 2 & 1 & $0 \cdot 5$ & 1 & 0.5 & 0.5 & 8 \\
\hline 8-con & 256 & 16 & 16 & 64 & 128 & 16 & 16 & 128 & 8 & 8 & $>128$ \\
\hline 8 -con* & 128 & 128 & 512 & 512 & 512 & 1024 & 128 & 256 & 128 & 32 & $>128$ \\
\hline NCTC 10499 & 128 & 128 & 512 & 128 & 512 & $>512$ & 128 & 512 & 32 & 16 & 512 \\
\hline 9-con & 256 & 256 & $>512$ & $>512$ & $>512$ & $>512$ & 512 & $>512$ & 512 & 64 & 512 \\
\hline 9-def & 8 & 0.5 & 128 & 16 & 128 & 64 & 128 & 128 & 4 & 4 & 128 \\
\hline NCTC 10257 & 128 & 128 & 256 & 32 & 128 & 64 & 128 & 64 & 16 & 4 & $>512$ \\
\hline 7-con & 256 & 256 & 1024 & 512 & 1024 & 512 & 512 & 512 & 128 & 32 & $>512$ \\
\hline 7-def & 16 & 2 & 64 & 16 & 128 & 8 & 32 & 4 & 2 & 2 & 128 \\
\hline 7-L1-def & 8 & 0.5 & 64 & 16 & 128 & 64 & 64 & 4 & 4 & 4 & 128 \\
\hline
\end{tabular}

IMP, imipenem; MEM, meropenem; AMP, ampicillin; PIP, piperacillin; CARB, carbenicillin; CEF, cefsulodin; CTX, cefotaxime; CTZ, ceftazidime ; CPZ, cefoperazone ; LMX, latamoxef; AZT, aztreonam.

mutant strains, 8-con and 8-con*, were obtained from strain NCTC 10258. Extracts of the latter mutant had about three-fold greater activity against both cefsulodin and imipenem than did those of the former.

All the basal mutant strains retained trace levels of $\beta$-lactamase activity but lost inducibility, suggesting that the mutations responsible for their behaviour were in regulatory genes rather than in the structural genes for the $\beta$-lactamases.

\section{Antibiotic susceptibility of inducibility mutant strains}

The MICs of various $\beta$-lactam agents for the parent and mutant strains determined on Mueller Hinton agar, are shown in table II. The MICs of the non- $\beta$ lactam agents that were tested-tetracycline, chloramphenicol and nalidixic acid-are not shown but remained within a range of one dilution for each series of mutant strains, which suggests that permeability had not been altered simultaneously with $\beta$-lactamase expression.

Of the three $\beta$-lactamase-inducible strains, NCTC 10258 was considerably the most susceptible to meropenem, piperacillin and various third-generation cephalosporins, whereas NCTC 10257 and NCTC 10499 were more resistant. These results apparently correlated with the lower enzyme levels inducible in strain NCTC 10258 (fig. 1). Mutant strains with highlevel $\beta$-lactamase constitutivity showed increased resistance to all antibiotics, except carbapenems, compared with $\beta$-lactamase-inducible strains. Mutant strain 8 -con*, with the higher enzyme activity, was more resistant than strain 8 -con. The $\beta$-lactamase basal mutant strains had 4-512-fold increased susceptibility to all antibiotics compared with the $\beta$ lactamase-inducible parent strains. Increased susceptibility was especially pronounced to meropenem and imipenem but was apparent also to penicillins and cephalosporins. Mutant strain 7-L1-def, which remained inducible for the L2 cephalosporinase, was as susceptible as strain 7-def (basal for both $\beta$-lactamases), except to cefsulodin, to which it remained as resistant as strain NCTC 10257.

\section{Effects of media on susceptibility and $\beta$-lactamase expression}

The parent and mutant strains appeared most resistant when tested on Mueller Hinton agar and least so on DST agar; they gave intermediate results on IsoSensitest agar (table III). These differences were least for imipenem, for which resistance was unequivocal on all media, and most evident for the cephalosporins and penicillins. For strain NCTC 10258 and its mutant strains, the MICs varied eight-fold, at most, according to the medium, but up to a 64-fold variation was observed for strains NCTC 10257, NCTC 10499 and their mutant strains.

Figs. $2 \mathrm{a}$ and $\mathrm{b}$ show induction of L1 $\beta$-lactamase by cefotaxime in $X$. maltophilia strains NCTC 10257 and NCTC 10499 during growth in Mueller Hinton broth, IsoSensitest broth and a broth medium equivalent to DST agar. The results showed that the level of induction obtained was independent of the growth medium.

\section{Discussion}

An initial aim was to obtain $X$. maltophilia mutant strains in which expression of only one or other of the $\beta$-lactamases was altered. However, it became apparent that the L1 and L2 enzymes shared overlapping regulatory systems. Thus, induction or mutations generally affected expression of both $\beta$-lactamases 
Table III. Ratios of MICs on DST and IsoSensitest agars to those on Mueller Hinton agar

\begin{tabular}{|c|c|c|c|c|c|c|c|c|c|c|c|c|c|c|}
\hline \multirow{3}{*}{ Strain } & \multicolumn{14}{|c|}{ Ratio* of MIC on Mueller Hinton agar to that on } \\
\hline & \multicolumn{7}{|c|}{ DST agar } & \multicolumn{7}{|c|}{ IsoSensitest agar } \\
\hline & IMP & MEM & I PIP & CEF & CTX & $\mathrm{CPZ}$ & LMX & IMP & MER & PIP & CEF & CTX & $\mathrm{CPZ}$ & LMX \\
\hline NCTC 10258 & $0 \cdot 25$ & 0.25 & 0.5 & 1 & 0.25 & 1 & $0 \cdot 5$ & 0.5 & 0.25 & 0.5 & 1 & 0.25 & 1 & 0.5 \\
\hline 8-con & $0 \cdot 12$ & $0 \cdot 25$ & 0.5 & 4 & $0 \cdot 12$ & 0.5 & $0 \cdot 12$ & 0.5 & 0.5 & 1 & 4 & 4 & 2 & 1 \\
\hline 8 -con* & 1 & 0.5 & 0.25 & 0.5 & $0 \cdot 5$ & $0 \cdot 25$ & 0.5 & 1 & 0.5 & 1 & 1 & 1 & 1 & 1 \\
\hline 8-def & 0.5 & 0.5 & 0.5 & 1 & 1 & 1 & 2 & 0.5 & $0 \cdot 25$ & 0.5 & 2 & 1 & 4 & 2 \\
\hline NCTC 10499 & 1 & 0.06 & 0.03 & 0.01 & 0.01 & 0.03 & 0.25 & 1 & 0.25 & 0.06 & 0.03 & 0.5 & 0.06 & 0.25 \\
\hline 9-con & 2 & 1 & $\mathrm{NC}$ & $\mathrm{NC}$ & 1 & 1 & 4 & 2 & 1 & $\mathrm{NC}$ & $\mathrm{NC}$ & 1 & 1 & 1 \\
\hline 9-def & 2 & 0.5 & 0.5 & $0 \cdot 12$ & 0.03 & 0.05 & 0.5 & 1 & 0.25 & 0.5 & 0.25 & 0.5 & 1 & 0.5 \\
\hline NCTC 10257 & 1 & $0 \cdot 25$ & $0 \cdot 25$ & 0.06 & 0.03 & $0 \cdot 25$ & 1 & 1 & 0.5 & 0.5 & 0.06 & 0.25 & 0.25 & 1 \\
\hline 7 -con & 0.5 & 0.5 & 0.06 & $0 \cdot 12$ & 0.03 & 0.06 & $0 \cdot 2$ & 0.5 & 0.5 & $0 \cdot 12$ & $0 \cdot 12$ & 0.25 & $0 \cdot 12$ & 0.25 \\
\hline 7-def & 0.25 & 1 & 1 & 1 & 0.25 & 2 & 2 & 0.25 & 0.5 & 1 & 1 & 0.25 & 2 & 2 \\
\hline 7-L1-def & 0.5 & 1 & 1 & 0.5 & 0.5 & 1 & 1 & 0.5 & 1 & 0.5 & 0.5 & 0.25 & 1 & 1 \\
\hline
\end{tabular}

Abbreviations, see footnote to table II. NC, not calculable; MICs on both media were above the highest concentration tested. When only one MIC was above the highest level tested, it was assumed that the actual MIC was twice that level.

* Values represent the MIC on the indicated medium (DST or IsoSensitest agar) divided by that on Mueller Hinton agar.

simultaneously, as demonstrated by the use of specific substrates and by iso-electric focusing of cell extracts. The overlap was not total, however, and for one mutant strain, 7-L1-def, expression of L1 $\beta$-lactamase became basal whilst that of L2 enzyme remained inducible.

The genetic basis for the linkage between expression of L1 and L2 enzymes can, at this stage, only be the subject of speculation. Both genes may have operatorpromoter regions that bind the same repressor or activator protein. Mutations that affect the production or structure of this protein, or of components, such as cell-wall precursors, that interact with it, may, in most cases, simultaneously regulate expression of both enzymes. It seems unlikely that the L1 and L2 genes are in the same operon and share a single operatorpromoter. The $\mathrm{L} 1$ gene has been cloned into plasmids, complete with considerable flanking sequences ${ }^{17}$ but these did not determine simultaneous production of L2 enzyme. Even less likely is the suggestion ${ }^{17}$ that a single structural gene encodes a monomeric $\beta$-lactamase precursor which is then assembled as a tetramer (L1 enzyme) or a dimer (L2 enzyme). L2 is not known to be dimeric and the two enzymes differ in their activity, substrate range and susceptibility to inhibition. Edetic acid is a strong inhibitor of the L1 enzyme but not of L2, ${ }^{5,6}$ whereas sulbactam and clavulanate give the reverse effect.

Our findings are consistent with the view that the enzymes contribute to, but are not wholly responsible for, the resistance of $X$. maltophilia to $\beta$-lactams. The L1 enzyme made a particularly large contribution to carbapenem resistance. Thus, mutant strains with basal expression of this enzyme, whether expression was inducible or basal for L2 $\beta$-lactamase, were exquisitely susceptible to meropenem (MIC $0.5 \mathrm{mg} / \mathrm{L}$ )
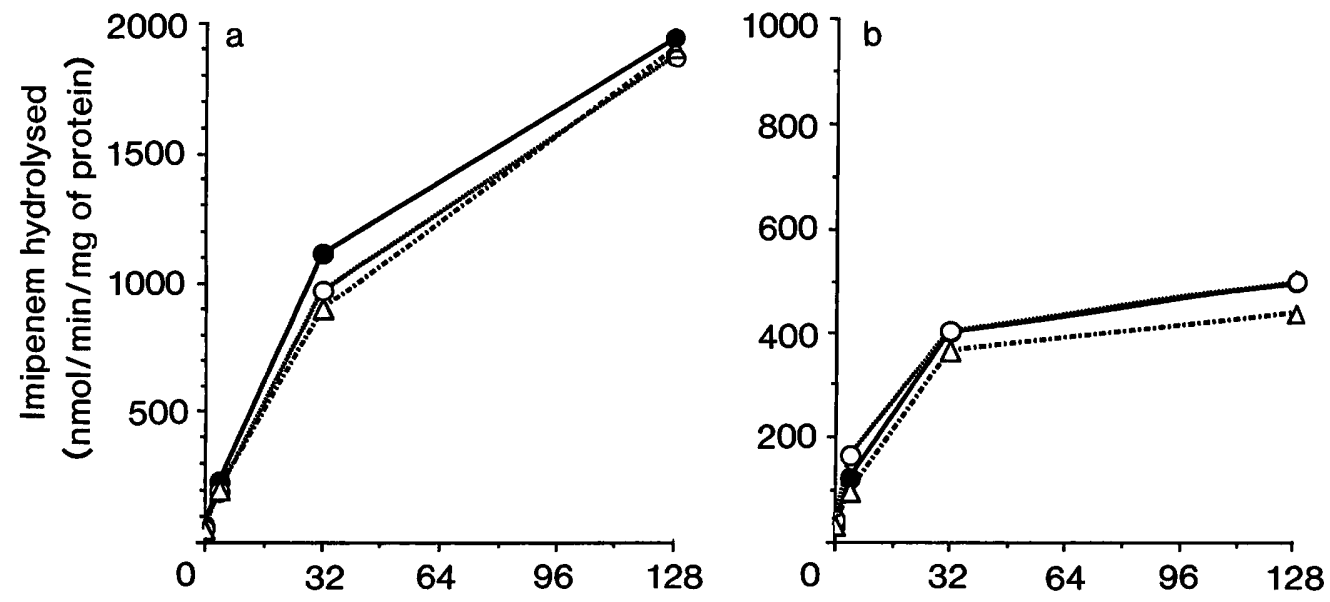

Cefotaxime concentration ( $\mathrm{mg} / \mathrm{L}$ )

Fig. 2. Induction of $\beta$-lactamase activity against imipenem by cefotaxime: (a) strain NCTC 10257 and (b) strain NCTC 10499 during growth in Mueller Hinton broth (O), IsoSensitest broth (O) and a broth medium equivalent to DST agar $(\triangle)$. Similar results were obtained with cefsulodin as the substrate. 
and moderately so to imipenem (MIC $4-16 \mathrm{mg} / \mathrm{L}$ ). Inducible $\beta$-lactamase expression gave some protection against third-generation cephalosporins, piperacillin and carbenicillin but constitutive hyperproduction (in mutant strains 7-con, 8-con, 8con* and 9-con) gave greater resistance. The retained inducibility of L2 enzyme, whilst L1 expression was basal, gave little protection in mutant strain 7-L1-def against any agents except cefsulodin. Protection from either inducible or constitutive $\beta$-lactamase expression was least against latamoxef, which is amongst the most stable agents to both enzymes. ${ }^{5,6}$

The ability of the inducibly expressed enzymes to determine resistance to many third-generation cephalosporins and ureidopenicillins contrasts with the behaviour seen with the inducible Class I $\beta$-lactamases typical of $P$. aeruginosa and some enterobacteria. The newer cephalosporins and ureidopenicillins are weak $\beta$-lactamase inducers for these organisms and the enzymes protect the bacteria only when they are constitutively hyperproduced, not while they remain inducible. ${ }^{13,18}$ This difference correlates with the fact that many of these agents, particularly cefotaxime, are much stronger $\beta$-lactamase-inducers for $X$. maltophilia than for $P$. aeruginosa and enterobacteria. ${ }^{7,18}$

Although the present data show that either inducible of high-level constitutive expression of L1 and L2 $\beta$ -

\section{References}

1. Muder RR, Yu VL, Dummer JS, Vinson C, Lumish RM. Infections caused by Pseudomonas maltophilia: expanding clinical spectrum. Arch Intern Med 1987; 147: 1672-1674.

2. von Graevenitz A. Ecology, clinical significance and antimicrobial susceptibility of infrequently encountered glucosenon-fermenting gram-negative rods. In: Gilardi G (ed) Nonfermentative gram-negative rods: laboratory identification and clinical aspects (Microbiology series 16). New York, Marcel Dekker Inc. 1985: 181-232.

3. Marshall WF, Keating MR, Anhalt JP, Steckelberg JM. Xanthomonas maltophilia: an emerging nosocomial pathogen. Mayo Clin Proc 1989; 64: 1097-1 104.

4. Rolston KVI, Anaissie EA, Bodey GP. In-vitro susceptibility of Pseudomonas species to fifteen antimicrobial agents. $J$ Antimicrob Chemother 1987; 19: 193-196.

5. Saino Y, Kobayashi F, Inoue M, Mitsuhashi S. Purification and properties of inducible penicillin $\beta$-lactamase isolated from Pseudomonas maltophilia. Antimicrob Agents Chemother 1982; 22: 564-570.

6. Saino Y, Inoue M, Mitsuhashi S. Purification and properties of an inducible cephalosporinase from Pseudomonas maltophilia GN12873. Antimicrob Agents Chemother 1984; 25: 362-365.

7. Mett H, Rosta S, Schacher B, Frei R. Outer membrane permeability and $\beta$-lactamase content in Pseudomonas maltophilia clinical isolates and laboratory mutants. Rev Infect Dis 1988; 10: 765-769.

8. Bicknell R, Emanuel EL, Gagnon J, Waley SG. The production and molecular properties of the zinc $\beta$-lactamase of Pseudomonas maltophilia IID 1275. Biochem J 1985; 229: 791-797. lactamases contributed to the insensitivity of $X$. maltophilia, they show also that these enzymes are not the only factors in the resistance of the organism. The basal mutant strains 7-def and 9-def retained substantial resistance to many penicillins and cephalosporins in spite of their loss of $\beta$-lactamase inducibility. The other basal mutant strain, 8 -def, was more susceptible, but had been derived from an unusuallysusceptible parent strain, which may inherently have been rather permeable to $\beta$-lactams.

In common with others, ${ }^{10,11}$ we found that $X$. maltophilia was more resistant to penicillins, cephalosporins and meropenem on Mueller Hinton agar than on DST or IsoSensitest agars. Imipenem resistance was not medium-dependent. These mediumdetermined effects were independent of the mode of expression of $\beta$-lactamase and occurred with the $\beta$ lactamase basal and high-level constitutive mutant strains as well as with their $\beta$-lactamase-inducible parent strains. These results, together with the finding that $\beta$-lactamase induction was equally efficient in all three media, discount $\beta$-lactamase as a source of the medium-dependent susceptibility in the species. It is possible that the growth medium modulates cell permeability and this is now being investigated.

We are grateful to ICI Pharmaceuticals for supporting this study.

9. Cartwright SJ, Waley SG. Purification of $\beta$-lactamases by affinity chromatography on phenylboronic acid-agarose. Biochem J 1984; 221 : 505-512.

10. Edwards JR, Turner PJ, Wannop C, Withnell ES, Grindey AJ, Nairn K. In-vitro antibacterial activity of SM-7338, a carbapenem antibiotic with stability to dehydropeptidase I. Antimicrob Agents Chemother 1989; 33: $215-222$.

11. King A, Boothman C, Phillips I. Comparative in-vitro activity of meropenem on clinical isolates from the United Kingdom. J Antimicrob Chemother 1989; 24 Suppl A: 3145.

12. Cullmann W, Dalhoff A, Dick W. Nonspecific induction of $\beta$ lactamase in Enterobacter cloacae. J Gen Microbiol 1984; 130: $1781-1786$.

13. Yang $Y$, Livermore DM, Williams RJ. Chromosomal $\beta$ lactamase expression and antibiotic resistance in Enterobacter cloacae. J Med Microbiol 1988; 25: 227-233.

14. Lowry OH, Rosebrough NJ, Farr AL, Randall RJ. Protein measurement with the Folin phenol reagent. $J$ Biol Chem 1951; 193: 265-275.

15. Matthew M, Harris AM, Marshall MJ, Ross GW. The use of analytical isoelectric focusing for detection and identification of $\beta$-lactamases. J Gen Microbiol 1975; 88: 169-178.

16. Yang Y. Chromosomal $\beta$-lactamase expression and antibiotic resistance in enterobacteria. PhD Thesis, University of London. 1990.

17. Dufresne J, Vézina G, Levesque RC. Molecular cloning and expression of the imipenem-hydrolyzing $\beta$-lactamase gene from Pseudomonas maltophilia in Escherichia coli. Rev Infect Dis 1988; 10: 806-817.

18. Livermore DM. Clinical significance of beta-lactamase-induction and stable derepression in gram-negative rods. Eur J Clin Microbiol 1987; 6: 439-445. 